\title{
Lesson of the month 2: IgG4-related renal mass with spontaneous resolution
}

\author{
Authors: Vijaya BP Ramasamy, ${ }^{A}$ Rhiannon Trefor, ${ }^{B}$ Kaushik Rajamani, ${ }^{C}$ Divya Santosh,, David Griffiths ${ }^{\mathrm{E}}$ \\ and Kieron Donovan ${ }^{\mathrm{F}}$
}

Immunoglobulin G4-related disease (IgG4-RD) is a systemic inflammatory condition that may involve any organ in the body, including the kidneys. However, renal parenchymal lesions are not seen frequently and the treatment strategy remains unclear. We describe a case of IgG4-related renal mass, which resolved spontaneously. The patient presented with right loin pain, constitutional symptoms and raised inflammatory markers. Magnetic resonance imaging (MRI) showed a large infiltrative mass centered on the right renal hilum and biopsy demonstrated histological changes in keeping with IgG4-RD. A careful 'watch-and-wait' approach was taken and at six months following initial presentation, the patient's symptoms had fully resolved and inflammatory markers had normalised. Repeat MRI showed almost complete resolution of the mass. We propose that a careful 'watchand-wait' approach could be considered as an alternative to immune suppression for IgG4-related renal masses, especially if they are not causing symptoms or organ compromise.

KEYWORDS: IgG4, renal tumour, IgG4-RD, inflammatory mass, storiform fibrosis

\section{Introduction}

Immunoglobulin G4-related disease (IgG4-RD) is a recently recognised systemic inflammatory condition characterised by mass-forming lesions, dense lymphoplasmacytic infiltrate rich in IgG4-positive plasma cells, storiform fibrosis and often, raised serum IgG4 levels. ${ }^{1}$

The aetiology and pathogenesis remain poorly understood, with autoimmunity and allergic-type reactions among the

Authors: ${ }^{A}$ SpR in nephrology, Nephrology and Transplant, University Hospital of Wales, Cardiff, UK; ${ }^{\mathrm{B}} \mathrm{SpR}$ in pathology, Pathology Department, University Hospital of Wales, Cardiff, UK; ${ }^{C}$ core medical trainee, Nephrology and Transplant, University Hospital of Wales, Cardiff, UK; DSpR in radiology, Radiology Department, University Hospital of Wales, Cardiff, UK; ${ }^{{ }^{2}}$ consultant pathologist, Pathology Department, University Hospital of Wales, Cardiff, UK; F consultant nephrologist, Nephrology and Transplant, University Hospital of Wales, Cardiff, UK postulated explanations. It was originally described as a cause of recurrent pancreatitis, but may also affect almost every other organ system.

Renal involvement, which is thought to be relatively uncommon, may present with a variety of clinical, biochemical, serological and radiological abnormalities. The most frequent manifestation is tubulointerstitial nephritis, but glomerular diseases like membranous nephropathy and membranoproliferative glomerulonephritis are also described in the literature. Solid, organised renal parenchymal lesions, that mimic malignancy, are recognised as a part of this clinical entity, but they are seen even less frequently. ${ }^{2}$ The optimal therapeutic strategies for IgG4-RD with renal involvement remain as yet unclear.

We describe the first documented case of an IgG4-related renal mass in the UK, and the first ever case of clinical and radiological spontaneous resolution of such a renal mass in the absence of any specific treatment.

\section{Case history}

A 37-year-old Caucasian man with no significant previous medical history, presented to the urologists with a few days of right-sided loin pain, night sweats and anorexia. He was apyrexial and had some mild right-lower quadrant abdominal tenderness.

His renal function and full blood count were normal, but his C-reactive protein (CRP) was elevated $(230 \mathrm{mg} / \mathrm{L}$; normal range, $<5 \mathrm{mg} / \mathrm{L}$ ). Abdominal ultrasound scan revealed a mildly enlarged right kidney that was suggestive of pyelonephritis. He was commenced on antibiotics and discharged home. Urine culture was subsequently reported negative for any microorganisms.

He presented to hospital with similar symptoms one month later. Blood tests once again revealed normal renal function and full blood count, and a raised CRP $(62 \mathrm{mg} / \mathrm{L})$. His urine culture was repeated and was negative for bacterial growth. Further radiological evaluation, including magnetic resonance imaging (MRI) showed a large infiltrative mass centred on the right renal hilum, which was encasing the renal vessels, inferior vena cava and proximal right ureter causing dilatation of the renal calyces. The initial clinical diagnosis was of a transitional cell carcinoma of the right kidney.

A radiologically guided renal biopsy was then performed, and histology showed interstitial expansion and diffuse inflammatory 

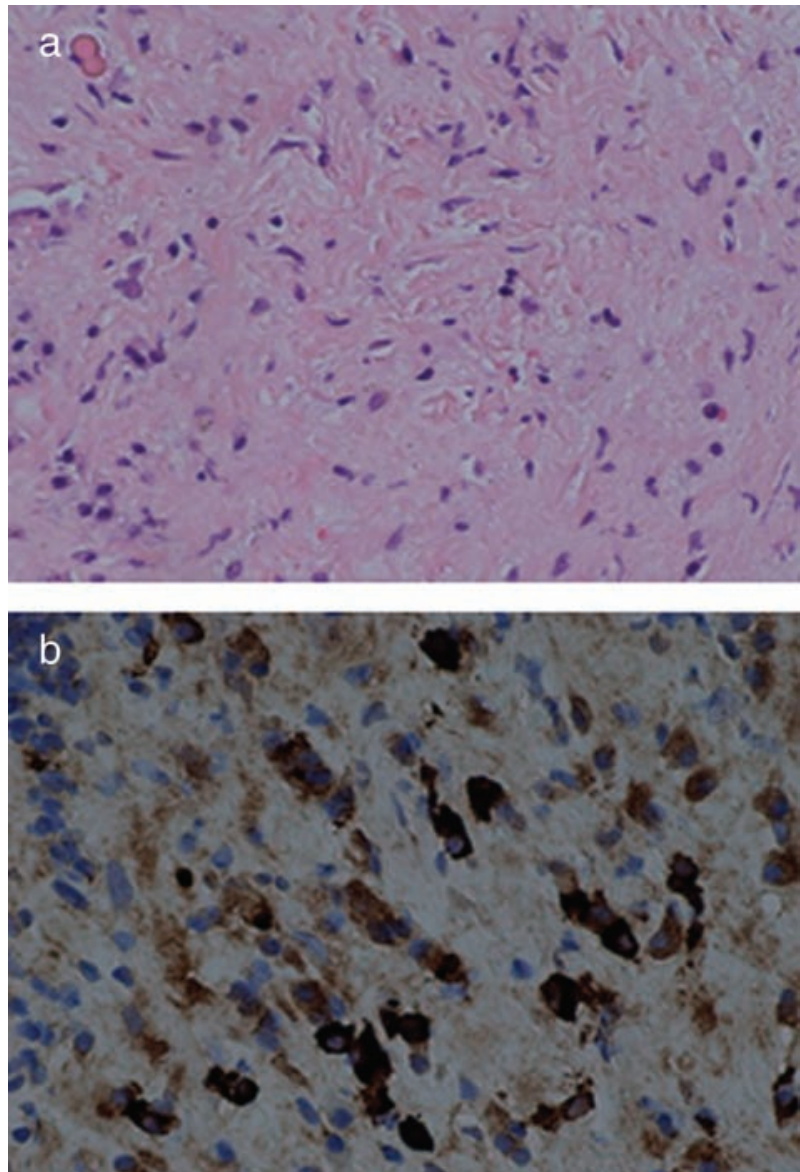

Fig 1. Renal biopsy histology. (a) H\&E stain (magnification, x20) shows storiform fibrosis with background lymphoplasmacytic infiltrate; (b) immunohistochemistry (magnification, $\times 40$ ) for IgG4 shows positive cytoplasmic staining in $>10$ plasma cells. $H \& E=$ hematoxylin and eosin; IgG4 = immunoglobulin G4

infiltrate consisting of lymphocytes, plasma cells and eosinophils. There was also evidence of cellular interstitial fibrosis with a focal storiform pattern, and immunohistochemistry for IgG4 showed $>10$ positive plasma cells per high power field (HPF) (Fig 1). There was no evidence of malignancy.

The patient was referred to nephrology outpatient services and was seen one month later. At this visit, he reported a significant and spontaneous improvement of his symptoms. His IgG level was raised at $19.70 \mathrm{~g} / \mathrm{L}(6.0-16.0)$ and IgG subtyping revealed an elevated IgG4 level at $2.53 \mathrm{~g} / \mathrm{L}(0.0-1.3)$. The diagnosis was discussed and, as his symptoms were improving, we undertook a careful 'watch-and-wait' approach with regular outpatient follow-up consultations and planned interval biochemical and radiological surveillance to monitor his clinical progress. Apart from a course of antibiotics prescribed during his initial presentation, he received no other treatment. After four months of follow up (six months after initial presentation), his renal function continued to be normal, IgG4 levels had fallen to normal $(0.75 \mathrm{~g} / \mathrm{L})$, and his CRP was undetectable. A follow-up MRI showed almost complete resolution of the right renal mass, with a very small residual area of scar tissue (Fig 2).
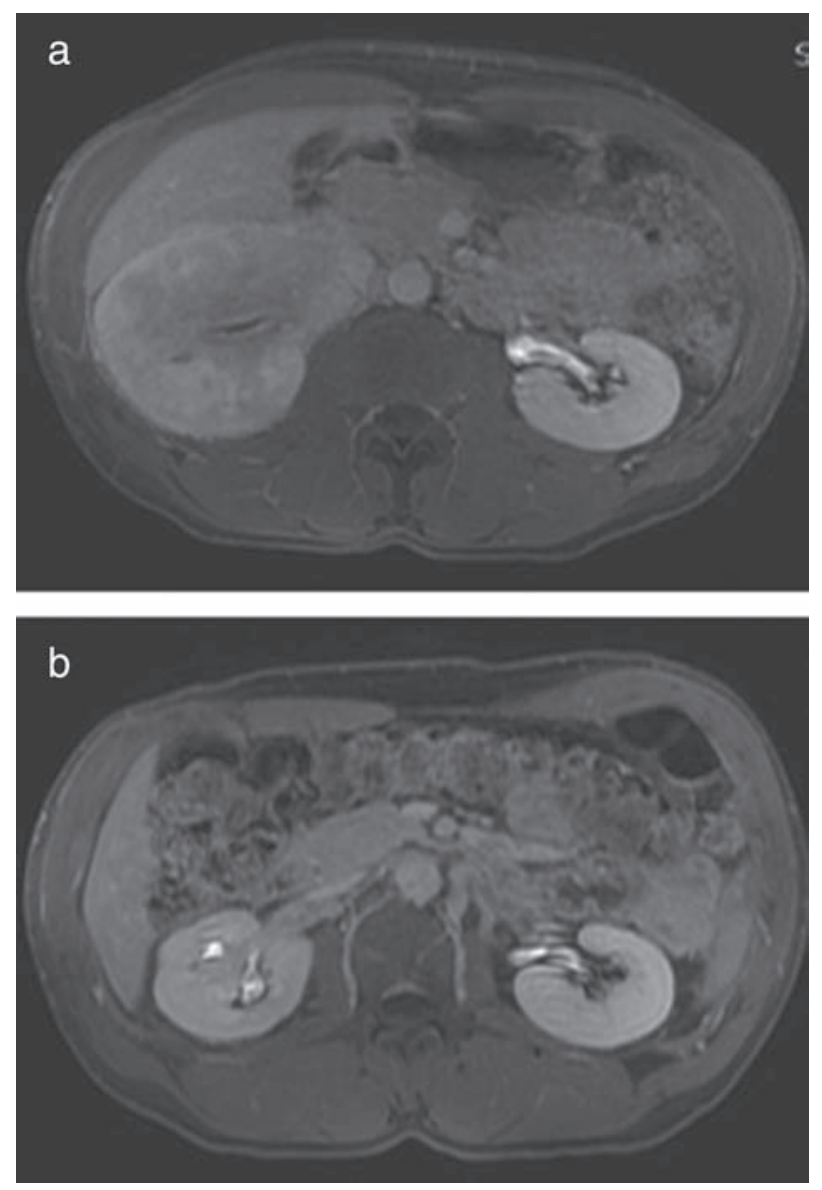

Fig 2. MRI of abdomen. (a) MRI with contrast at presentation; (b) MRI with contrast at six months. MRI = magnetic resonance imaging.

\section{Discussion}

Development of an isolated renal mass is an uncommon presentation of IgG4-RD and, in fact, has not been previously reported in the UK to our knowledge.

Previously, there have been reports of such lesions being mistaken for malignancies that have even led to nephrectomy, with a post surgical histological diagnosis of IgG4-RD., Our case highlights the need for appropriate and optimal investigations for any renal mass, especially those with accompanying clinical or biochemical features suggestive of an ongoing inflammatory process, before definite surgery is undertaken. We propose in such cases that, in addition to radiological imaging with MRI or computed tomography scan, serum immunoglobulin levels are measured. If IgG levels are raised, then this should merit IgG subtyping. Although only about $80 \%$ of patients with IgG4-RD have raised IgG4 levels, this proportion rises significantly in IgG4-RD patients with renal involvement, with some case series showing that all such patients have raised IgG4 levels. ${ }^{7}$

Although there are no internationally accepted diagnostic criteria for renal IgG4-RD, Kawano et al proposed a method based on the presence of typical radiological appearances, raised serum IgG4 levels, specific histological findings and 
kidney damage, to generate a diagnostic label of either definite, probable or possible renal IgG4-RD. ${ }^{7}$

The Consensus statement on the pathology of IgG-RD informs that 'the histological diagnosis of IgG4-RD rests on the combined presence of the characteristic histological appearance of dense lymphoplasmacytic infiltrate, storiform-type fibrosis or obliterative phlebitis and increased number of IgG4-positive plasma cells', which in the case of renal IgG4-RD, should be $>10$ cells per HPF in a renal biopsy tissue. ${ }^{8}$

The treatment of IgG4-RD is contentious, and there is no randomised controlled trial data. The indications for treatment, the choice of drugs and duration of therapy are all matters of opinion. Glucocorticoids have been used the most, but evidence of efficacy is based on small, retrospective, and observational studies. One glucocorticoid treatment approach is by initiating prednisolone between $0.6-1.0 \mathrm{mg} / \mathrm{kg}$ daily as an induction treatment for $2-4$ weeks, following which the dose is gradually weaned down to $5 \mathrm{mg}$ every 1-2 weeks based on clinical response. At 2-3 months, the prednisolone is either stopped or continued at a low dose. ${ }^{9,10}$

The roles of other immune suppressants, including azathioprine, mycophenolate mofetil, methotrexate and B-cell depleting agents, such as rituximab, are largely unproven, though they may have some theoretical merit. Watchful waiting has also been advocated in literature, but this is generally for asymptomatic, indolent cases with no vital organ involvement. In our patient this approach was adopted as he demonstrated spontaneous symptomatic and inflammatory marker improvement.

\section{Conclusion}

Renal IgG4-RD should be considered as a differential diagnosis for unusual renal mass lesions, and investigated against the diagnostic criteria proposed by Kawano et al. A careful 'watchand-wait' approach could be considered as an alternative to immune suppression for IgG4-related renal masses, especially if they are not causing symptoms or organ compromise, and if there are features suggesting systemic improvement.

\section{References}

1 Stone J, Zen Y, Deshpande V. IgG4-related disease. N Engl J Med 2012;366:539-51.

2 Saeki T, Nishi S, Imai N et al. Clinicopathological characteristics of patients with IgG4-related tubulointerstitial nephritis. Kidney Int 2010;78:1016-23.

3 Alkhasawneh AN, Allan RW. IgG4 inflammatory pseudotumor of the kidney. Case Rep Urol 2012;2012:919087.

4 Nofuji S, Shindo T, Mizuno T et al. A case of IgG4-related kidney disease mimicking a renal cell carcinoma. Hinyokika Kiyo 2013;59:513-5.

5 Sah RP, Chari ST. Serologic issues in IgG4-related systemic disease and autoimmune pancreatitis. Curr Opin Rheumatol 2011;23:108-13.

6 Tabata T, Kamisawa T, Takuma K et al. Serum IgG4 concentrations and IgG4-related sclerosing disease. Clin Chim Acta 2009;408:25-8.

7 Kawano M, Saeki T, Nakashima H et al. Proposal for diagnostic criteria for IgG4-related kidney disease. Clin Exp Nephrol 2011;15:615-26.

8 Deshpande V, Zen Y, Chan J KC et al. Consensus statement on the pathology of IgG4-related disease. Mod Pathol 2012;25:1181-92.

9 Shimosegawa T, Chari ST, Frulloni L et al. International consensus diagnostic criteria for autoimmune pancreatitis. Int J Pancreatol 2011;40:352-8.

10 Kamisawa T, Shimosegawa T, Okazaki K et al. Standard steroid treatment for autoimmune pancreatitis. Gut 2009;58:1504-7.

Address for correspondence: Dr VBP Ramasamy, Nephrology and Transplant, University Hospital Wales, Cardiff CF14 4XW, UK.

Email: vijaya.ramasamy@wales.nhs.uk 